\title{
Heroin delay discounting and impulsivity: Modulation by DRD1 genetic variation
}

\author{
Tabitha E.H. Moses $^{1}$ | Margit Burmeister ${ }^{2}$ | Mark K. Greenwald ${ }^{1}$
}

\author{
${ }^{1}$ School of Medicine, Wayne State University, \\ Detroit, Michigan \\ ${ }^{2}$ University of Michigan, Ann Arbor, Michigan \\ Correspondence \\ Mark K. Greenwald, Department of Psychiatry \\ and Behavioral Neurosciences, Tolan Park \\ Medical Building, 3901 Chrysler Service Drive, \\ Suite 2A, Detroit, MI 48201, USA. \\ Email: mgreen@med.wayne.edu \\ Funding information \\ National Institute on Drug Abuse, Grant/ \\ Award Number: R01 DA015462
}

\begin{abstract}
Background: Dopamine D1 receptors (encoded by DRD1) are implicated in drug addiction and high-risk behaviors. Delay discounting (DD) procedures measure decisional balance between choosing smaller/sooner rewards vs larger/later rewards. Individuals with higher DD (rapid discounting) are prone to maladaptive behaviors that provide immediate reinforcement (eg, substance use). DRD1 variants have been linked with increased DD (in healthy volunteers) and opioid abuse. This study determined whether four dopaminergic functional variants modulated heroin DD and impulsivity.
\end{abstract}

Methods: Substance use, DD, and genotype data (DRD1 rs686 and rs5326, DRD3 rs6280, COMT rs4680) were obtained from 106 current heroin users. Subjects completed an array of DD choices during two imagined conditions: heroin satiation and withdrawal. Rewards were expressed as $\$ 10$ heroin bag units, with maximum delayed amount of 30 bags. Delays progressively increased from 3 to 96 hours.

Results: $\quad D R D 1$ rs686 (A/A, $n=25 ; \mathrm{G} / \mathrm{A}, \mathrm{n}=56 ; \mathrm{G} / \mathrm{G}, \mathrm{n}=25$ ) was linearly related to the difference in heroin DD (area under the curve; $A U C$ ) between the heroin satiation and withdrawal conditions; specifically, G/G homozygotes had a significantly smaller (satiation minus withdrawal) AUC difference score had higher drug-use impulsivity questionnaire scores, relative to $\mathrm{A} / \mathrm{A}$ homozygotes, with $\mathrm{G} / \mathrm{A}$ intermediate. DRD3 and COMT variants were not associated with these DD and impulsivity outcomes.

Conclusion: DRD1 rs686 modulated the difference in heroin DD score between pharmacological states and was associated with drug-use impulsivity. These data support a role of DRD1 in opioid DD and impulsive behaviors.

\section{KEYWORDS}

delay discounting, dopamine, DRD1, heroin, impulsivity

\section{1 | INTRODUCTION}

Delay discounting (DD) procedures measure the decisional balance between choosing a smaller/sooner reward versus a larger/later reward ${ }^{1}$; selecting a smaller reward sooner (steeper discounting) is associated with extent of impulsive behavior in that situation. ${ }^{2}$ Individuals with steeper discounting are prone to maladaptive behaviors (eg, substance use). ${ }^{3}$ Relative to healthy controls, heroin users choose more immediate reinforcers. ${ }^{4}$ Traditional discounting methods assess DD over relatively long intervals (eg, months to years), which may not be ecologically relevant for a person using heroin every day. ${ }^{1}$ We previously examined heroin DD as a pharmacological-state measure among regular heroin users, employing a condensed time scale to make the paradigm more relevant to real-world drug use. ${ }^{5}$ The 
previous within-subject study found that DD was dramatically steeper during an imagined opioid withdrawal than satiation condition. In the satiation condition, individuals with lower IQ and higher drug-use impulsivity scores exhibited steeper heroin discounting. ${ }^{5}$

Previous studies have found that DD with nondrug goods is modulated by dopamine genetic variation and these variations have been associated with high-risk behaviors, including substance use., ${ }^{3,6-8}$ Although most opioid pharmacogenetic studies focus on opioid receptor mutations, opioid use indirectly stimulates dopamine, thus functional dopamine genetic variants could also relate to opioid-use behaviors. ${ }^{9,10}$ Several studies have explored the role of dopamine D2 receptors in chronic substance use, ${ }^{11-13}$ but minimal research has focused on more acute decision-making in substance use and D1 receptor specifically.

The D1 receptor is a G-protein coupled receptor primarily expressed in the striatum, and has several functional single nucleotide polymorphisms (SNPs) ${ }^{14}$ D1 receptors have been implicated in multiple brain functions including reward and reinforcement. ${ }^{15-17}$ Although both D1 and D2 receptors are associated with decision-making and reward, the varying roles and locations of these receptors result in different effects. For example, within the prefrontal cortex (PFC), pharmacological studies suggest D2 receptors are involved in flexible decision making (eg, making a new choice when the likelihood of loss of reward is high) whereas D1 receptors are involved in maintaining a choice (eg, making the same choice despite a high likelihood of loss of reward). ${ }^{18}$ This complementary role for D1 and D2 receptors is also seen in learning paradigms. Research suggests D1 receptors are involved with learning to initiate actions whereas D2 receptors are involved with learning to inhibit actions. ${ }^{14,19}$ D1 receptor density or activation in certain brain regions (eg, insular cortex, PFC, and thalamus) also modulates impulsive decision-making, ${ }^{20-22}$ and systemic administration of a D1 antagonist increased preference for a smallersooner reward over a larger-later reward, ie, impulsive choice. ${ }^{20,23}$

Some studies have found DRD1 variants to be linked with alcohol and tobacco use disorders (reviewed by $^{24}$ ) and with altered DD in healthy volunteers. ${ }^{3}$ To our knowledge, only one published study has related DRD1 polymorphisms to behavioral variation among regular opioid users, finding an association with rate of progression to heroin dependence in a Chinese Han sample. ${ }^{25} \mathrm{~A}$ literature review suggested four different functional SNPs relevant to dopaminergic activity and acute decision-making: two DRD1 SNPs (rs686 and rs5326), ${ }^{15,26}$ DRD3 rs6280, ${ }^{7,27}$ and Catechol-O-methyltransferase (COMT) rs4680. ${ }^{27,28}$ DRD3 rs6280 was included because it has been previously associated with several types of substance use/dependence (see review by Le Foll et $\mathrm{al}^{24}$ ) and this specific variant is associated with aberrant decision making ${ }^{29}$ and with modulating response to reward. ${ }^{30}$ We included COMT in our analyses because it affects dopamine concentrations in the PFC, an area strongly implicated in rewardrelated decision-making. ${ }^{31}$

The present candidate gene study is aimed to determine whether dopamine-system functional variants modulated heroin DD and drug-use impulsivity. We hypothesized that dopamine genetic variation would be related to DD. On the basis of findings of our previous work on DD in heroin users, we also hypothesized that dopamine genetic variation would be associated with IQ and drug-use impulsivity scores. ${ }^{5}$

\section{2 | MATERIALS AND METHODS}

\section{1 | Participants}

This study used screening data from four laboratory studies approved by the Institutional Review Boards at Wayne State University and the University of Michigan, registered under clinical trials NCT00218309, NCT00218361, NCT00608504, and NCT00684840. Participants were recruited via print media advertisements and word-of-mouth referral from the Detroit, Michigan Metropolitan Area. Nontreatment-seeking individuals between 18 and 55 years of age who used heroin regularly (at least weekly) and denied any major medical or psychiatric disorders during an initial phone screening were invited for an in-person visit. Participants were eligible to complete the in-person battery if they tested positive for opioid use (more than $300 \mathrm{ng} / \mathrm{mL}$ ), negative for alcohol (less than $0.002 \%$; Alco Sensor III Breathalyzer), and were cognitively intact (total IQ score greater than or equal to 80 on the Shipley Institute of Living Scale ${ }^{32}$ ).

\section{2 | Genotyping}

Blood samples were collected and participant DNA was extracted using the Qiagen kit (formerly Gentra Puregene). The Golden Gate drug addiction Illumina panel ${ }^{33}$ was used to genotype the blood samples. For this analysis, we focused on dopamine-system genetic variants: DRD1 rs686 [3'UTR; G/A] and rs5326 [5'UTR; G/A], DRD3 rs6280 [missense; C/T, Ser ${ }^{9} \mathrm{Gly}$ ], and COMT rs4680 [missense; G/A, $\left.\mathrm{Val}^{158} \mathrm{Met}\right]$.

\section{3 | Phenotyping}

Substance use characteristics were measured using the Drug History and Use Questionnaire (available on request). As previously described, ${ }^{5}$ delay discounting measures were obtained during two within-subject, experimental conditions: imagined heroin satiation and withdrawal. The order of these two imagined conditions was randomized for each participant. Rewards were expressed as $\$ 10$ heroin bag units (21 decreasing values from 30 to 0.3 bags), with seven increasing delays $(3,6,12,24,48,72$, and 96 h) to a delayed amount of 30 bags. This task was chosen as it allowed us to focus on the innovative question as to whether a simulated pharmacological-state condition alters heroin DD. As this is a novel task there are no data on its test-retest reliability; however, studies show that traditional monetary DD measures and some variations on the task (eg, sexual discounting) are reliable in retesting conditions. ${ }^{34-36}$ Drug-use impulsivity was measured with the 30-item Impulsive Relapse Questionnaire (IRQ) which, unlike indices of trait impulsivity, specifically measures impulsivity as it relates to using drugs (eg, impulsive choice to purchase more 
drugs) $)^{37}$ using five subscales: Automaticity, Speed (to return to use), Control Deficit, Denial, and Capacity for Delay. Ancestral race was measured by self-report. We previously used ancestry informative markers to confirm self-reported racial identity in this sample. ${ }^{38}$

\section{4 | Data analyses}

Area under the curve (AUC) was used to measure DD, as it was normally distributed and well suited for ANOVA and correlations. ${ }^{39}$ Initial analyses used repeated measures mixed-model analysis of covariance (ANCOVA) with genotype as the between-subjects factor (separate analysis for each genotype), pharmacological-state condition (eg, heroin satiation vs withdrawal) as within-subject factor and race and IQ as covariates. For any significant effects, we also conducted stepwise linear regression analyses, controlling for IQ, which explained variance in our earlier study ${ }^{5}$ and race because it is an important factor in genetic studies. ${ }^{40,41}$ We chose to conduct ANCOVA as the primary analysis with secondary linear regression because ANCOVA provides information about the initial significant interactions, whereas the subsequent regression allows us to more thoroughly consider the potential contributions of other covariates. Following initial analyses, a DD difference score was calculated from the difference between the AUC in each imagined condition. This difference score was used as a simple way to display the statistical interactions between genotype and condition and represents pharmacological sensitivity to the shift from satiation to withdrawal.

Within each genotype, we measured the allelic distribution to check for adequate group sizes and covaried for race and IQ because of our prior findings. ${ }^{5}$ All descriptive data are presented as mean \pm one standard deviation. All analyses were conducted with SPSS v. 25 and used the criterion of $P<.05$ to reject the null hypothesis. We include DD effect size estimates using partial eta-squared $\left(\eta^{2}\right)$.

\section{3 | RESULTS}

\section{1 | Participant characteristics}

We obtained complete data from 106 heroin-using participants. The average participant was a $42.7 \pm 10.0$-year-old African American (55.7\%) male (72.6\%) with $12.2 \pm 1.4$ years of education and a Shipley estimated IQ score of $104.4 \pm 10.8$. On average, participants had been using heroin for $20.4 \pm 12.6$ years and initiated heroin use at $22.4 \pm 6.5$ years old.

We obtained results for DRD1 rs5326 but did not analyse this variant further as minor allele frequency was too low. Using an online calculator, http://www.oege.org/software/cubex/, ${ }^{42}$ we found DRD1 rs686 was in high linkage disequilibrium with rs5326 but not substitutable $\left(r^{2}=0.2114 ; D^{\prime}=1.00\right)$. As expected (ie, because DRD1, DRD3, and COMT are located on different chromosomes), rs686 was not in LD with DRD3 rs6280 $\left(r^{2}=0.0175 ; D^{\prime}=0.141\right)$ or COMT rs4680 $\left(r^{2}=0.0174 ; D^{\prime}=0.179\right)$. Table 1 shows genotype and allelic frequencies for DRD1 rs686 for African Americans ( $n=59$ ), Caucasians ( $n=47)$, and overall sample $(N=106)$. Using an online calculator, http://www.oege.org/software/hardy-weinberg.html, ${ }^{43}$ genotype frequencies for rs686 did not deviate significantly from the HardyWeinberg equilibrium (HWE) in the overall samples or in the African American or Caucasian subsamples ( $p s>0.05$ ). Allelic frequencies did not differ significantly by race $\left(x^{2}=5.51 ; P=.064\right)$, therefore, we conducted analyses in the combined sample. Tables 2 and 3 show genotype and allelic distributions for the other two SNPs, which did not deviate from HWE.

TABLE 1 DRD1 rs686 genotype distributions and allele frequencies

\begin{tabular}{llllllll} 
DRD1 rs686 (N) & A/A & G/A & G/G & A allele & G allele & HWE $\chi^{2}$ & P \\
Black (59) & $12(20.3 \%)$ & $28(47.5 \%)$ & $19(32.2 \%)$ & $52(44.1 \%)$ & $66(55.9 \%)$ & 0.08 & .775 \\
White (47) & $13(27.7 \%)$ & $28(59.6 \%)$ & $6(12.8 \%)$ & $54(57.4 \%)$ & $40(42.6 \%)$ & 2.24 & .134 \\
Overall (106) & $25(23.6 \%)$ & $56(52.8 \%)$ & $25(23.6 \%)$ & $106(50 \%)$ & $106(50 \%)$ & 0.34 & .560 \\
\hline
\end{tabular}

TABLE 2 DRD3 rs6280 genotype distributions and allele frequencies

\begin{tabular}{lclclrrr} 
DRD3 rs6280 (N) & A/A & A/G & G/G & A allele & G allele & HWE $\chi^{2}$ & P \\
Black (59) & $5(8.5 \%)$ & $24(40.7 \%)$ & $30(50.8 \%)$ & $34(28.8 \%)$ & $84(71.2 \%)$ & 0.004 & .949 \\
White (47) & $21(44.7 \%)$ & $23(48.9 \%)$ & $3(6.4 \%)$ & $65(69.1 \%)$ & $29(30.9 \%)$ & 1.02 & .314 \\
Overall (106) & $26(24.5 \%)$ & $47(44.3 \%)$ & $33(31.1 \%)$ & $99(46.7 \%)$ & $113(53.3 \%)$ & 1.27 & .260 \\
\hline
\end{tabular}

TABLE 3 COMT rs4680 genotype distributions and allele frequencies

\begin{tabular}{llllllrl} 
COMT rs4680 (N) & A/A & A/G & G/G & A allele & G allele & HWE $\chi^{2}$ & P \\
Black (58) & $4(6.9 \%)$ & $24(41.4 \%)$ & $30(51.7 \%)$ & $32(27.6 \%)$ & $84(72.4 \%)$ & 0.07 & .786 \\
White (47) & $8(17.0 \%)$ & $26(55.3 \%)$ & $13(27.7 \%)$ & $42(44.7 \%)$ & $52(55.3 \%)$ & 0.67 & .414 \\
Overall (105) & $12(11.4 \%)$ & $50(47.6 \%)$ & $43(41.0 \%)$ & $74(35.2 \%)$ & $136(64.8 \%)$ & 0.20 & .657 \\
\hline
\end{tabular}




\section{2 | Delay discounting}

Heroin DD overall (ie, averaged across pharmacological-state condition) did not significantly differ for any of the genotypes examined. There was no main effect of genotype on DD score in either the satiation condition $\left(F_{2,101}=0.44, P=.643, \eta^{2}=0.009\right)$ or the withdrawal condition $\left(F_{2,101}=2.05, P=.134, \eta^{2}=0.039\right)$. This indicates that the DD score was not significantly different across genotypes in either condition. We did find a significant interaction of DRD1 rs686 (G/G $\mathrm{n}=25 ; \mathrm{G} / \mathrm{A}, \mathrm{n}=56 ; \mathrm{A} / \mathrm{A}, \mathrm{n}=25)$ and condition, $\mathrm{F}_{2,101}=3.66$ $P=.029, \eta^{2}=0.068$ (Figure 1) after controlling for race and IQ, indicating there is a significant relationship between genotype and the change in response to imagined conditions in this DD paradigm. After discovering an interaction effect and no simple main effects, we computed the (satiation minus withdrawal) AUC difference score to reflect change in DD between the two conditions (Figure 2). This difference score is used as a marker of pharmacological sensitivity as it shows the change in DD response between the two conditions. We observed an allelic dose-effect: the $G / G$ group had a significantly greater change in $\mathrm{DD}$ between conditions than the A/A group, and the heterozygous (G/A) group was intermediate and did not significantly differ from either homozygote group. DRD1 rs686 remained significantly related to the change in DD, $F_{2,101}=3.66, P=.029$, when controlling for IQ, $F_{1,101}=15.89, P<.001, \eta^{2}=0.136$, and race, $F_{1,101}=5.34$, $P=.023, \eta^{2}=0.050$. To ensure the differences we found were related to differences in discounting between the two conditions rather than alternative confounding factors, we also examined mean indifference points per group per discounting state (Figure 3). This analysis showed that from the 12-hour time delay onward, there is a stable difference in the change score illustrating that the significant interaction effect is because of the alteration in discount rate (ie, grows with delay to heroin receipt) rather than other confounding factors.

We conducted a stepwise linear regression analysis, again controlling for race and IQ, to explore the predictive effect of DRD1 rs686 genotype on the heroin DD difference score. The final model (step 3) included IQ ( $\left.\beta=.351 ; t=3.91, P<.001, \Delta r^{2}=0.113\right)$, DRD1 rs686 ( $\left.\beta=-.224 ; t=-2.46, P=.016, \Delta r^{2}=0.035\right)$, and race $\left(\beta=-.200 ; t=-2.18, P=.032, \Delta r^{2}=0.038\right)$ and was significant overall, $F_{2,105}=7.74, P<.001$, explaining $16.1 \%$ of total adjusted variance in the DD difference score. This finding shows that IQ, genotype, and race all independently play significant roles in the difference score between the two imagined conditions in heroin DD.

\section{3 | Other phenotypes}

We observed no significant genotype differences for any demographic characteristics (Table 4) but again controlled for race. DRD1 rs686 A/A homozygotes (vs G/G, with G/A intermediate) had higher IRQ speed scores. DRD3 and COMT variants were unrelated to impulsivity outcomes (ie, IRQ scores).
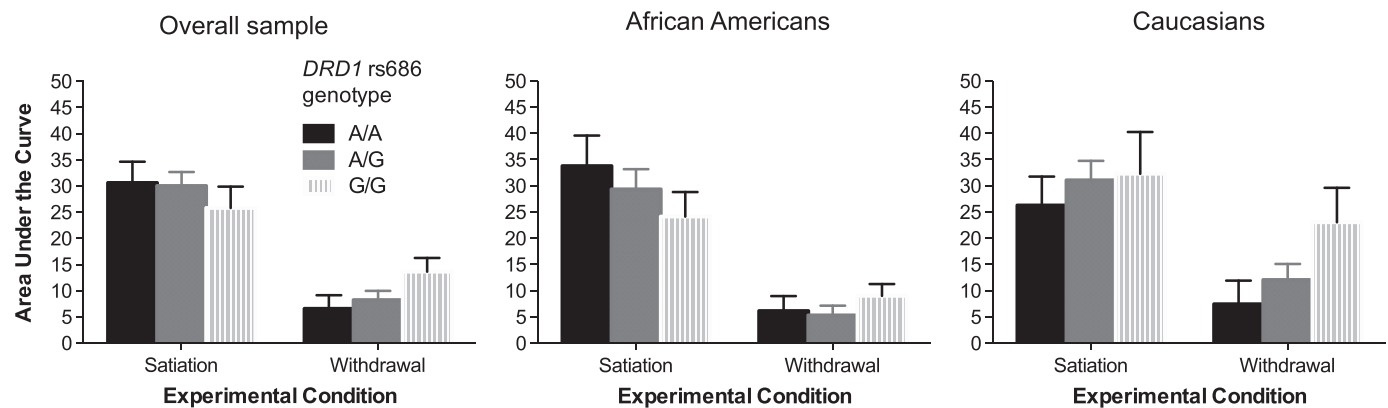

FIGURE 1 Mean (1 SEM) heroin delay-discounting area-under-the-curve (AUC) scores during participant-imagined satiation and withdrawal pharmacological-state conditions, by DRD1 rs686 genotype and race

Overall sample

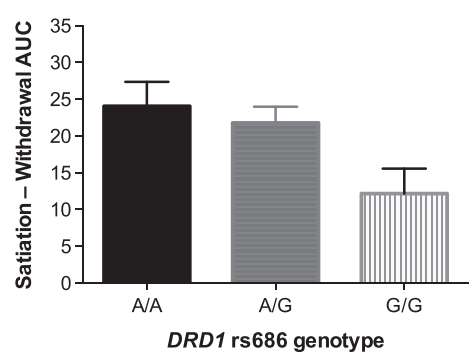

African Americans

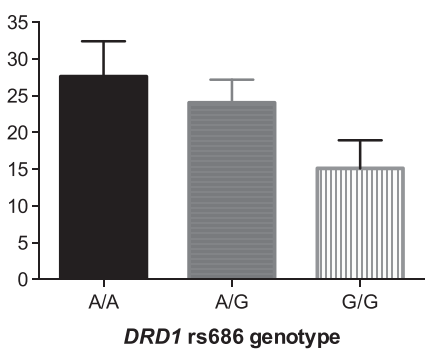

Caucasians

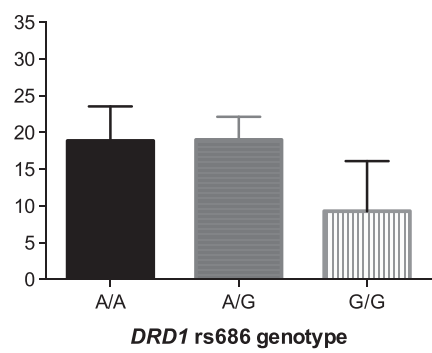

FIGURE 2 Mean (1 SEM) change in heroin delay-discounting area-under-the-curve (AUC) scores between pharmacological-state conditions, by DRD1 rs686 genotype and race 
$\mathrm{A} / \mathrm{A}$

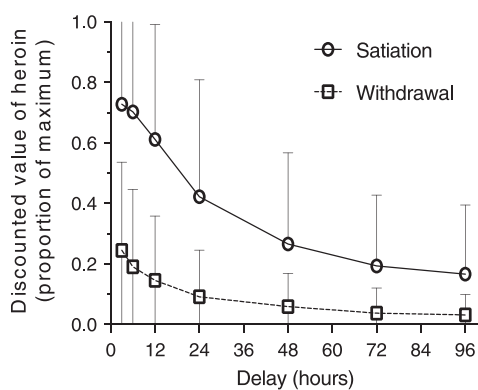

$\mathrm{A} / \mathrm{G}$

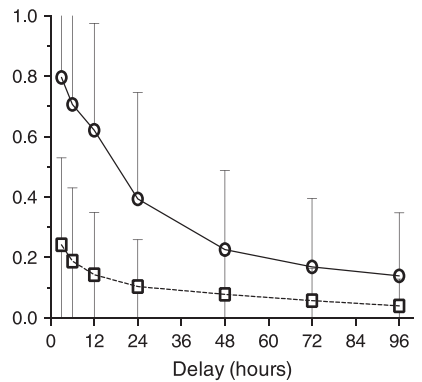

$\mathrm{G} / \mathrm{G}$

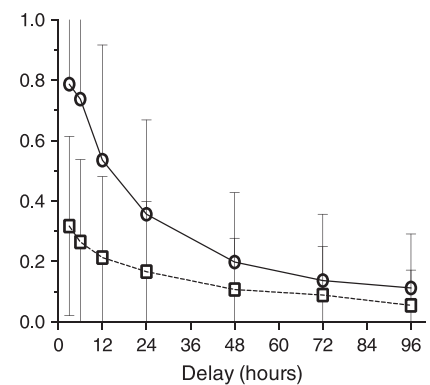

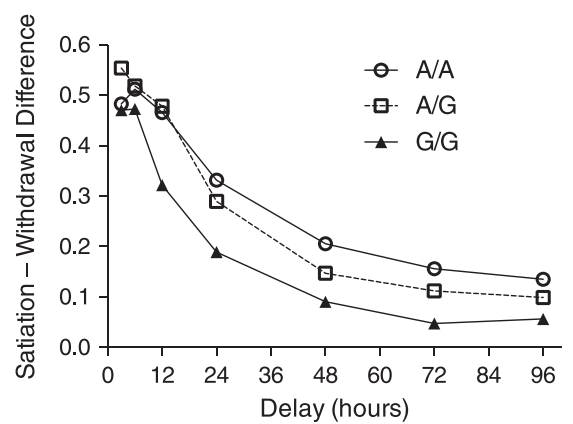

FIGURE 3 Mean (1 SEM) heroin delay-discounting indifference points for satiation and withdrawal conditions as a function of DRD1 rs686 genotype (upper row) and time course of (satiation-withdrawal) difference score for each genotype group (lower panel)

TABLE 4 Demographic and substance use characteristic variation for DRD1 rs686 genotypes in the overall sample

\begin{tabular}{|c|c|c|c|c|c|}
\hline & $\mathrm{A} / \mathrm{A}(n=25)$ & $\mathrm{G} / \mathrm{A}(n=56)$ & $\mathrm{G} / \mathrm{G}(n=25)$ & $\chi^{2} / F$ & $P$ \\
\hline Sex (male) & $76.0 \%(19)$ & $69.6 \%(39)$ & $76.0 \%(19)$ & 0.54 & .764 \\
\hline Age (years) & $41.4 \pm 9.3$ & $41.8 \pm 10.5$ & $46.1 \pm 9.0$ & 1.89 & .156 \\
\hline Current injection drug use & $68.0 \%(17)$ & $58.9 \%(33)$ & $44.0 \%(11)$ & 3.04 & .219 \\
\hline IRQ speed ${ }^{\pi}$ & $9.6 \pm 3.5^{b}$ & $11.4 \pm 3.6^{\mathrm{ab}}$ & $12.3 \pm 4.1^{a}$ & 3.47 & .035 \\
\hline Number of heroin quit attempts & $9.7 \pm 19.6$ & $12.0 \pm 22.5$ & $10.7 \pm 20.1$ & 0.14 & .868 \\
\hline
\end{tabular}

"Shared superscripts indicate non-significant differences between group means in post hoc tests.

\section{4 | DISCUSSION}

The present study examined how dopaminergic genetic variation impacted ecologically-relevant (brief time-scale) heroin DD under different simulated pharmacological conditions and drug-use impulsivity among out-of-treatment heroin users. We studied functional polymorphisms implicated in opioid use disorder that are related to dopaminergic activity and acute decision-making: DRD1 rs686, ${ }^{15,24,26}$ DRD3 rs6280, ${ }^{7,27}$ and COMT rs4680. ${ }^{27,28}$ We hypothesized that dopaminergic genetic variation would be associated with heroin $\mathrm{DD}, \mathrm{IQ}$, and drug-use impulsivity. ${ }^{5}$ Although none of these dopamine geneticvariants was associated with overall rates of heroin DD under satiation and withdrawal conditions, DRD1 rs686 was uniquely associated with the change in heroin discount rate between satiation and withdrawal conditions.
Among these heroin users, DRD1 rs686 genotype (after controlling for IQ and race) explained $4.2 \%$ of variance in the change in discounting between pharmacological-state conditions. There was an allelic dose-effect: $G / G$ homozygotes discounted heroin more than $A / A$ homozygotes or $A / G$ heterozygotes in the satiation condition and comparatively less in the withdrawal condition, resulting in a significantly smaller change in AUC between the two conditions (Figure 2). We theorize that the DD difference score reflects sensitivity to the dynamic shift between opioid satiation vs withdrawal (use vs abstinence) states that these habitual heroin users regularly encounter. Thus, A/A homozygotes exhibited greater sensitivity to changes in simulated pharmacological state (ie, larger shift in heroin DD from satiation to withdrawal) than G/G homozygotes. This genotype group difference in heroin discounting was not evident at 3and 6-hour delays, but was larger and consistent from the 12-hr to 
96-hr time period measured (Figure 3). We did not find any main effect between DRD1 rs686 genotype and DD. We developed the difference score in response to the significant interaction effect between genotype and condition. The finding of this interaction effect in the absence of a main effect suggests this genotype plays a larger role in sensitivity to change in opioid agonist stimulation level (as measured by the DD change score) than discounting itself. Taken together, our findings suggest that DRD1 genotype modulates pharmacological state-dependent (satiation minus withdrawal) change in heroin discounting, an effect that grows with delay to receipt of heroin.

Although demographic and substance-use characteristics (Table 4) did not significantly differ by DRD1 rs686 genotype, there was an allelic dose-effect for IRQ Speed subscale scores. This aligns with our previous findings that higher IRQ speed scores were associated with greater heroin DD. ${ }^{5}$ We did not find an association between IQ and DRD1 genotype. We postulated this relationship based on our observations that lower IQ was associated with more impulsive drug choices $^{5}$; the lack of association here suggests the IQ/impulsivity relationship may be separate from the one we see between dopaminergic genetic variation and drug-use impulsivity.

The biological impact of DRD1 rs686 plausibly relates to these behavioral findings. Previous studies found the A-allele is associated with increased DRD1 gene expression and D1 receptor density ${ }^{19,44}$ relative to G-allele carriers, which may partly explain differences in pharmacological-state heroin discounting in this study. A-allele carriers have higher D1 receptor density and thus might be more sensitive to dynamic state-dependent changes in dopamine that occur during drug satiation and withdrawal (which can happen on a daily basis for heroin users). Previous research has demonstrated results that support this theory of increased sensitivity. One study found that individuals with A-alleles were most likely to continue to experience opioid-induced euphoria even after chronic use. ${ }^{45}$ This contrasts with G-allele carriers who may have decreased D1 receptor expression, possibly resulting in their overall higher rates of discounting and decreased difference score. This theory is supported by the fact that systemic administration of a D1 antagonist has been shown to increase discounting. ${ }^{20,23}$ This theory is also enhanced by our finding that individuals with Galleles had a higher IRQ speed score indicating increased drug use impulsivity. Additionally, G-allele carriers have been shown to exhibit more depressive symptoms than A-allele carriers. ${ }^{46}$ Depressive symptoms are associated with impairments in emotion regulation and cognitive control, ${ }^{47}$ which may decrease sensitivity to pharmacologicstate changes among G-allele carriers.

The present study has several limitations. First, substance-use variables were self-reported, which may introduce recall bias. Second, the DD paradigm required participants to imagine heroinsatiated and withdrawal conditions without specific guidance; yet, there is no direct means to confirm they imagined the correct scenario. Despite this concern, the present findings align with prior work using this paradigm in opioid-dependent individuals, ${ }^{48}$ which suggests consistency in the imagined conditions. Third, we have a relatively small sample size, which may have resulted in a lack of power. Fourth, we used nonstandard experimental conditions for this study. Both the relatively short delay times and the within-subjects comparison of the two conditions and the absence of a monetary DD task, are not standard relative to the existing literature, which may influence the relevance of our findings. Fifth, although we did collect information about major medical and psychiatric conditions, we excluded these covariates in the analyses because of concerns of confounding and multiple comparisons. Unfortunately, this also means that our findings are less generalizable because co-occurring conditions are common among this population. Sixth, we used selfreported race/ethnicity rather than ancestral informative markers and although these two measures are highly correlated, ${ }^{49}$ genetic markers are more specific. ${ }^{41}$ However, our prior use of ancestral markers to confirm self-reported race in this sample, ${ }^{38}$ and our finding that race was not significantly associated with DRD1 rs686 allelic variation, reduces the impact of this potential limitation. Seventh, this study is a candidate gene study, which comes with its own limitations. ${ }^{50}$ These analyses were based on a priori assumptions about the genes and pathways in question and it is possible that our underlying assumptions are incorrect. Furthermore, although these findings align with our hypotheses of the mechanisms underlying these differences, this study design does not allow us to know whether the candidate gene in question is the causal mechanism for this observation.

Our findings indicate an important relationship between the DRD1 gene and acute drug-use decision-making and impulsive behaviors. We found DRD1 modulated the shift in heroin, discounting between the two pharmacological-state conditions and was associated with drug-use impulsivity. These data support a role of DRD1 in opioid DD and impulsive drug-use behaviors. DRD1 variants could be useful markers for understanding impulsive behaviors in opioid-dependent individuals. The use of behavioral measures has proved integral in our understanding of substance use, and pharmacogenetic analyses of these phenotypes could improve our mechanistic understanding and approaches to addiction treatment.

\section{ACKNOWLEDGEMENTS}

NIH grant R01 DA015462 (to M.K.G.) from the National Institute on Drug Abuse, Helene Lycaki/Joe Young, Sr. Funds (State of Michigan), and the DetroitWayneMental HealthAuthoritysupportedthis research. Funding sources had no role in the design or conduct of this study, nor in the preparation of this manuscript. The authors thank Ken Bates for recruiting participants and Lark Haunert, Debra Kish, Joi Moore, Melissa Williams, Elorie Eggleston, Hayley Harrison, and Lisa Sulkowski for data collection and management, and Ela Sliwerska and the University of Michigan DNA core for genotyping.

\section{CONFLICT OF INTEREST}

All authors declare no conflict of interest with respect to the conduct or content of this work. 


\section{AUTHORS CONTRIBUTION}

T.E.H.M. completed analyses and drafted the manuscript. M.B. oversaw genotyping analysis and data interpretation, and edited the manuscript. M.K.G. oversaw all aspects of the project including data collection and management, analyses, and co-wrote the manuscript.

\section{ORCID}

Mark K. Greenwald (10) https://orcid.org/0000-0002-9541-7321

\section{REFERENCES}

1. Bickel WK, Marsch LA. Toward a behavioral economic understanding of drug dependence: delay discounting processes. Addiction. 2001;96(1):73-86.

2. Peters J, Büchel C. The neural mechanisms of inter-temporal decisionmaking: understanding variability. Trends Cogn Sci. 2011;15(5):227-239.

3. Lin H, Epstein LH. Living in the moment: effects of time perspective and emotional valence of episodic thinking on delay discounting. Behav Neurosci. 2014;128(1):12-19.

4. Li X, Zhang F, Zhou Y, Zhang M, Wang X, Shen M. Decision-making deficits are still present in heroin abusers after short- to long-term abstinence. Drug Alcohol Depend. 2013;130(1-3):61-67.

5. Stoltman JJK, Woodcock EA, Lister JJ, Lundahl LH, Greenwald MK. Heroin delay discounting: modulation by pharmacological state, druguse impulsivity, and intelligence. Exp Clin Psychopharmacol. 2015;23(6):455-463.

6. Anokhin AP, Grant JD, Mulligan RC, Heath AC. The genetics of impulsivity: evidence for the heritability of delay discounting. Biol Psychiatry. 2015;77(10):887-894

7. Gray JC, MacKillop J. Impulsive delayed reward discounting as a genetically-influenced target for drug abuse prevention: a critical evaluation. Front Psychol. 2015;6:1104.

8. Kohno M, Nurmi EL, Laughlin CP, et al. Functional genetic variation in dopamine signaling moderates prefrontal cortical activity during risky decision making. Neuropsychopharmacology. 2016;41(3):695-703.

9. Spanagel R, Weiss F. The dopamine hypothesis of reward: past and current status. Trends Neurosci. 1999;22(11):521-527.

10. Steidl S, Wasserman DI, Blaha CD, Yeomans JS. Opioid-induced rewards, locomotion, and dopamine activation: a proposed model for control by mesopontine and rostromedial tegmental neurons. Neurosci Biobehav Rev. 2017;83:72-82.

11. Belcher AM, Volkow ND, Moeller FG, Ferré S. Personality traits and vulnerability or resilience to substance use disorders. Trends Cogn Sci. 2014;18(4):211-217.

12. Dobbs LK, Lemos JC, Alvarez VA. Restructuring of basal ganglia circuitry and associated behaviors triggered by low striatal $\mathrm{d} 2$ receptor expression: implications for substance use disorders. Genes Brain Behav. 2017;16(1):56-70.

13. O'Hara BF, Smith SS, Bird G, et al. Dopamine d2 receptor rflps, haplotypes and their association with substance use in black and caucasian research volunteers. Hum Hered. 1993;43(4):209-218.

14. Kravitz AV, Kreitzer AC. Striatal mechanisms underlying movement, reinforcement, and punishment. Phys Ther. 2012;27:167-177.

15. Batel P, Houchi H, Daoust M, Ramoz N, Naassila M, Gorwood P. A haplotype of the drd1 gene is associated with alcohol dependence. Alcohol Clin Exp Res. 2008;32(4):567-572.

16. Beninger RJ, Miller R. Dopamine d1-like receptors and reward-related incentive learning. Neurosci Biobehav Rev. 1998;22(2):335-345.
17. Luca P, Laurin N, Misener VL, et al. Association of the dopamine receptor d1 gene, drd1, with inattention symptoms in families selected for reading problems. Mol Psychiatry. 2007;12(8):776-785.

18. Jenni NL, Larkin JD, Floresco SB. Prefrontal dopamine d1 and d2 receptors regulate dissociable aspects of decision making via distinct ventral striatal and amygdalar circuits. J Neurosci. 2017;37(26):6200-6213.

19. Baetu I, Burns NR, Urry K, Barbante GG, Pitcher JB. Commonly-occurring polymorphisms in the comt, drd1 and drd2 genes influence different aspects of motor sequence learning in humans. Neurobiol Learn Mem. 2015;125:176-188.

20. Pattij T, Schetters D, Schoffelmeer ANM. Dopaminergic modulation of impulsive decision making in the rat insular cortex. Behav Brain Res. 2014;270:118-124.

21. Sonntag KC, Brenhouse HC, Freund N, Thompson BS, Puhl M, Andersen SL. Viral over-expression of $\mathrm{d} 1$ dopamine receptors in the prefrontal cortex increase high-risk behaviors in adults: comparison with adolescents. Psychopharmacology (Berl). 2014;231(8):1615-1626.

22. Wang Z, Liang S, Yu S, et al. Distinct roles of dopamine receptors in the lateral thalamus in a rat model of decisional impulsivity. Neurosci Bull. 2017;33(4):413-422.

23. Koffarnus MN, Newman AH, Grundt P, Rice KC, Woods JH. Effects of selective dopaminergic compounds on a delay discounting task. Behav Pharmacol. 2011;22(4):300-311.

24. Le Foll B, Gallo A, Le SY, et al. Genetics of dopamine receptors and drug addiction: a comprehensive review. Behav Pharmacol. 2009;20(1):1-17.

25. Peng S, Du J, Jiang $\mathrm{H}$, et al. The dopamine receptor d1 gene is associated with the length of interval between first heroin use and onset of dependence in chinese han heroin addicts. I Neural Transm. 2013;120(11):1591-1598.

26. Jacobs MM, Ökvist A, Horvath M, et al. Dopamine receptor d1 and postsynaptic density gene variants associate with opiate abuse and striatal expression levels. Mol Psychiatry. 2012;18:1205.

27. Verdejo-García A, Lawrence AJ, Clark L. Impulsivity as a vulnerability marker for substance-use disorders: review of findings from high-risk research, problem gamblers and genetic association studies. Neurosci Biobehav Rev. 2008;32(4):777-810.

28. Kreek MJ, Nielsen DA, Butelman ER, LaForge KS. Genetic influences on impulsivity, risk taking, stress responsivity and vulnerability to drug abuse and addiction. Nat Neurosci. 2005;8(11):1450-1457.

29. Rajan R, Krishnan S, Sarma G, Sarma SP, Kishore A. Dopamine receptor d3 rs6280 is associated with aberrant decision-making in parkinson's disease. Mov Disord Clin Pract. 2018;5(4):413-416.

30. Savitz J, Hodgkinson CA, Martin-Soelch C, et al. The functional drd3 ser9gly polymorphism (rs6280) is pleiotropic, affecting reward as well as movement. PLoS One. 2013;8(1):e54108.

31. Yuferov V, Levran O, Proudnikov D, Nielsen DA, Kreek MJ. Search for genetic markers and functional variants involved in the development of opiate and cocaine addiction and treatment. Ann N Y Acad Sci. 2010;1187(1):184-207.

32. Zachary RA. The manual of the shipley institute of living scale. 120th ed. Los Angeles, CA: Western Psychological Services; 1991.

33. Hodgkinson CA, Yuan Q, Xu K, et al. Addictions biology: haplotypebased analysis for 130 candidate genes on a single array. Alcohol Alcohol. 2008;43(5):505-515.

34. Beck RC, Triplett MF. Test-retest reliability of a group-administered paper-pencil measure of delay discounting. Exp Clin Psychopharmacol. 2009;17(5):345-355.

35. Johnson MW, Bruner NR. Test-retest reliability and gender differences in the sexual discounting task among cocaine-dependent individuals. Exp Clin Psychopharmacol. 2013;21(4):277-286. 
36. Odum AL. Delay discounting: trait variable? Behav Processes. 2011;87(1):1-9.

37. Adinoff B, Rilling LM, Williams MJ, et al. Impulsivity, neural deficits, and the addictions. J Addict Dis. 2007;26(sup1):25-39.

38. Woodcock EA, Lundahl LH, Burmeister M, Greenwald MK. Functional mu opioid receptor polymorphism (oprm1 a $118 \mathrm{~g}$ ) associated with heroin use outcomes in caucasian males: a pilot study. Am J Addict. 2015;24(4):329-335.

39. Myerson J, Green L, Warusawitharana M. Area under the curve as a measure of discounting. J Exp Anal Behav. 2001;76(2):235-243.

40. Burchard EG, Ziv E, Coyle N, et al. The importance of race and ethnic background in biomedical research and clinical practice. N Engl J Med. 2003;348(12):1170-1175.

41. Yaeger R, Avila-Bront A, Abdul K, et al. Comparing genetic ancestry and self-described race in African Americans born in the United States and in Africa. Cancer Epidemiol Biomarkers Prev. 2008;17(6):1329-1338.

42. Gaunt TR, Rodríguez S, Day INMN. Cubic exact solutions for the estimation of pairwise haplotype frequencies: implications for linkage disequilibrium analyses and a web tool 'cubex. BMC Bioinformatics. 2007;8(1):428.

43. Rodriguez S, Gaunt TR, Day INMM. Hardy-weinberg equilibrium testing of biological ascertainment for mendelian randomization studies. Am J Epidemiol. 2009;169(4):505-514.

44. Huang W, Li MD. Differential allelic expression of dopamine d1 receptor gene (drd1) is modulated by microrna mir-504. Biol Psychiatry. 2009;65(8):702-705.
45. Zhu F, Yan C, Wen Y, et al. Dopamine d1 receptor gene variation modulates opioid dependence risk by affecting transition to addiction. PLoS One. 2013;8(8):e70805.

46. Jiménez KM, Pereira-morales AJ, Forero DA. A functional polymorphism in the drd1 gene, that modulates its regulation by mir-504, is associated with depressive symptoms. Psychiatry Investig. 2018;15(4):402-406.

47. Joormann J, Gotlib IH. Emotion regulation in depression: relation to cognitive inhibition. Cogn Emot. 2010;24(2):281-298.

48. Giordano L, Bickel W, Loewenstein G, Jacobs E, Marsch L, Badger G. Mild opioid deprivation increases the degree that opioid-dependent outpatients discount delayed heroin and money. Psychopharmacology (Berl). 2002;163(2):174-182.

49. Tang H, Quertermous $T$, Rodriguez B, et al. Genetic structure, selfidentified race/ethnicity, and confounding in case-control association studies. Am J Hum Genet. 2005;76(2):268-275.

50. Daly AK, Day CP. Candidate gene case-control association studies: advantages and potential pitfalls. $\mathrm{Br} J$ Clin Pharmacol. 2001;52(5):489-499.

How to cite this article: Moses TEH, Burmeister $M$, Greenwald MK. Heroin delay discounting and impulsivity: Modulation by DRD1 genetic variation. Addiction Biology. 2020;25:e12777. https://doi.org/10.1111/adb.12777 